\title{
Aulas, publicidad y ficción televisiva: una experiencia de innovación docente
}

\author{
Marta PACHECo RuEdA \\ Universidad de Valladolid \\ martapr@ega.uva.es
}

Recibido: 06/10/2012

Aceptado: 23/01/2013

\begin{abstract}
Resumen
El presente artículo muestra el desarrollo y resultados de un proyecto de innovación docente, concebido para hacer uso de los textos audiovisuales no sólo como herramienta pedagógica sino también como espoleta de una toma de postura comprometida y responsable con la realidad social. La novedad de la propuesta estriba en la utilización de la ficción televisiva y, en concreto, la serie Mad Men, en el marco de la docencia universitaria de la Publicidad, orientada a un manejo crítico de la información, que promueva la reflexión de los estudiantes en torno al fenómeno publicitario en el contexto socioeconómico actual.

Palabras clave: Innovación docente, Mad Men, publicidad, educomunicación, competencias

\section{Classrooms, Advertising and Television Fiction: an Experience of Innovative Teaching}

\begin{abstract}
This paper shows the development and results of an innovative teaching project, conceived to make use of audiovisual texts not only as a pedagogic tool but also as a committed and responsible approach to social reality. The novelty of the proposal is the use of television fiction and, in particular, Mad Men serial, within the framework of university teaching of Advertising, targeting to a critical information management, and to promote the reflection of the students on the advertising phenomenon in the current socioeconomic context.
\end{abstract}

Keywords: Innovative teaching, Mad Men, advertising, media literacy, competencies

\section{Referencia normalizada}

PACHECO RUEDA, Marta (2013): “Aulas, publicidad y ficción televisiva: una experiencia de innovación docente”. Estudios sobre el Mensaje Periodístico. Vol. 19, Núm. especial marzo, págs.: 361-369. Madrid, Servicio de Publicaciones de la Universidad Complutense.

Sumario: 1. Introducción. 2. Fundamentación teórica del proyecto. 3. Desarrollo. 4. Resultados y valoración del proyecto. 5. Referencias bibliográficas.

\section{Introducción}

El proyecto de innovación docente «Aprender con $\mathrm{Mad}$ Men $\rangle^{1}$ fue desarrollado en el marco de los estudios del Grado en Publicidad y Relaciones Públicas de la Universidad de Valladolid durante los cursos académicos 2010/11 y 2011/12. El proyecto estaba orientado a la adquisición de diversas competencias establecidas para Teoría e Historia de la Publicidad. La serie de televisión Mad Men, que explora la historia de una agencia de publicidad en los años 60 en EEUU, constituía una pieza fundamental de dicho proyecto. Producida por Lionsgate y creada por Mathew Wiener, la serie

1 Dicho proyecto fue seleccionado en la convocatoria 2011 de Proyectos de Innovación Docente para el EEES de la Universidad de Valladolid y estuvo dirigido por Pilar San Pablo Moreno, profesora asociada del Área de Comunicación Audiovisual y Publicidad de la Universidad de Valladolid durante los cursos académicos 2005/06 a 2011/12. 
ha sido emitida por el canal AMC de televisión por cable en EEUU y por otras cadenas en todo el mundo desde 2007.

Múltiples premios de la industria audiovisual avalan el prestigio de la serie. Además, Mad Men se ha convertido en un producto icónico muy relevante y es considerada por numerosos teóricos del cine y la publicidad como un referente que marca estilo y tendencias en estos ámbitos, así como en el de la moda. La serie está siendo objeto de estudio desde el ámbito académico con la publicación de monografías en que, con un enfoque multidisciplinar, se analiza dicha serie. Así mismo, Mad Men constituye un ejemplo paradigmático de las nuevas ficciones televisivas que abren espacios para la creación audiovisual, explorando territorios que el cine tradicional todavía no ha podido alcanzar. Dado que las series expanden el imaginario contemporáneo y crean nuevas redes por las que circulan sus propuestas narrativas, se convierten en material didáctico sumamente útil como herramienta docente.

A la hora de concebir y diseñar el proyecto, tomamos como referentes teóricos las aportaciones de destacados autores dentro del ámbito de la educomunicación, movimiento que comenzó a desarrollarse durante la segunda mitad del siglo XX en diferentes contextos geográficos con el propósito de introducir en la enseñanza primaria y secundaria el análisis, estudio y prácticas de los medios de comunicación.

\section{Fundamentación teórica del proyecto}

La educomunicación implica la interrelación de dos campos de estudio: la educación y la comunicación. Como señala Aparici (2010: 17), «la educomunicación nos presenta una filosofía y una práctica de la educación y la comunicación basadas en el diálogo y en la participación, que no requieren sólo de tecnologías sino de un cambio de actitudes y de concepciones pedagógicas y comunicativas». Muchos de sus principios pedagógicos están basados en la comunicación dialógica planteada por Paulo Freire, para quien el diálogo constituye un elemento crucial para problematizar el conocimiento: no se trata de un diálogo complaciente ni una mera conversación, sino de una modalidad que indaga en los saberes mutuos y cuestiona el conocimiento preestablecido, una metodología y una filosofía. Para Freire, la relación entre los interactuantes en el proceso de la educación y de la comunicación debe responder a un acto de encuentro y de reciprocidad, dando lugar a un proceso de toma de conciencia crítica tanto de docentes como de alumnos: «El diálogo y la problematización no adormecen a nadie. Concientizan. En la dialoguicidad, en la problematización, educador-educando y educando-educador desarrollan ambos una postura crítica, de la cual resulta la percepción de que todo este conjunto de saber se encuentra en interacción. Saber que refleja al mundo y a los hombres, en el mundo y con él, explicando al mundo, pero sobre todo justificándose en su transformación» (Freire citado por Aparici, 2010: 13).

Freire pone en tela de juicio el modelo tradicional de enseñanza-aprendizaje, basado en unas relaciones rígidas entre docentes y alumnos, que dan lugar a un proceso de comunicación unidireccional, en el que el alumno asume el rol de pasivo receptor de conocimientos. Por su parte, Kaplún hace hincapié en que tal modelo desvaloriza la experiencia de vida de los alumnos y aboga por la superación de ese modelo tradi- 
cional unidireccional por otro bidireccional: «La apropiación del conocimiento por parte de los educandos se cataliza cuando se los instituye y potencia como emisores. Su proceso de aprendizaje se ve favorecido e incrementado por la realización de productos comunicables y efectivamente comunicados. Si educar es involucrar en un proceso de múltiples interacciones, un sistema será tanto más educativo cuanto más rica sea la trama de flujos comunicacionales que sepa abrir y poner a disposición de los educandos» (Kaplún, 2010: 60-61).

Mucho se ha escrito acerca del gran poder de comunicación y la influencia de los medios audiovisuales, en general, y del cine, en particular. Compartiendo plenamente este planteamiento, nos detendremos aquí en destacar cómo el cine-y, por extensión, la ficción televisiva-, puede ser utilizado no sólo como un entretenimiento sino también como una herramienta formativa. Sacar al cine del espacio reservado al entretenimiento para otorgarle en las aulas un rango similar al de los manuales teóricos que recogen el saber por escrito, esto es, habilitarlo como «una herramienta pedagógica permanente» (Pereira y Valle, 2002: 236) requiere de un alumnado capacitado en el manejo de un alfabeto visual, con su gramática, su sintaxis y su narratividad específicas. Ahora bien, que los alumnos decodifiquen en el arte cinematográfico la información, depende de sus propias habilidades para reconocer y decodificar, a su vez, las emociones que el propio cine pone en movimiento en ellos. Como apunta FERRÉS (2010: 53), «las pantallas tradicionales ofrecen, en fin, y sobre todo, la oportunidad para una constante liberación emotiva. Los programas, concebidos siempre como espectáculo (los informativos se rigen por los parámetros expresivos del show business), prenden fuertemente en el ánimo del espectador, que se implica emocionalmente en ellos mediante los mecanismos psíquicos de la identificación y la proyección».

Una auténtica autoridad emocional se adquiere cuando no sólo se es capaz de reconocer e identificar cómo somos afectados por las emociones sino, además, cómo éstas ponen en movimiento decisiones y actitudes específicas que colaboran determinantemente en la construcción de nuestra imagen del mundo. Tal y como afirma Maturana, «no es la razón lo que nos lleva a la acción, sino la emoción» (Maturana, 1999, citado por Ferrés, 2010: 263). De aquí se desprende que sólo analizando nuestra experiencia emocional ante las pantallas podemos descubrir cómo nos afectan y que sólo recurriendo a la experiencia emocional de los interlocutores podemos provocar cambios en ellos (Ferrés, 2010: 263).

De la imagen que tenemos del mundo y de nosotros dentro de ese mundo, depende la calidad de nuestra vida. Por eso el cine, en tanto que nos habla en imágenes, colabora en la configuración de ese universo personal y social. Por ello, la propuesta pasa por afrontar el reto de reconocer que el cine no será ya más «un parche alternativo dentro del proceso de enseñanza-aprendizaje del alumnado, sino como una porción integrante de todo el currículo» (Coll et al., 1995). El cine posee una innegable influencia en los valores sociales y «proporciona a la educación el método intuitivo adecuado para afianzar contenidos nuevos» (Pereira y Marín, 2001: 235).

Si tenemos en cuenta las tesis de Bauman acerca de los actuales tiempos líquidos en los que «las formas sociales [...] ya no pueden mantener su consistencia porque se descomponen y derriten antes de que se cuente con el tiempo necesario para asumir- 
las [...], la virtud que se proclama más útil para servir a los intereses individuales no es la conformidad a las normas (que, en cualquier caso, son escasas, y a menudo contradictorias) sino la flexibilidad: la presteza para cambiar de tácticas y estilos en un santiamén, para abandonar compromisos y lealtades sin arrepentimiento, y para ir en pos de las oportunidades según la disponibilidad del momento, en vez de seguir las propias referencias consolidadas» (Bauman, 2007: 7-11), la labor del docente en el marco de la educomunicación, consiste en trazar sendas adecuadas para conocer y reconocer en el cine el reflejo de esa realidad, que conduce a la producción y reproducción de cierto orden cultural, reflexivo y, por qué no decirlo, también crítico.

Conviene aprovechar esa faceta en la cual el cine es susceptible de convertirse en algo que forma, en algo que educa, en algo que invita a hacerse consciente, y recordar el concepto que de cine formativo defiende de la Torre: «[...] cine formativo es la emisión y recepción intencional de películas portadoras de valores culturales, humanos, técnico-científicos o artísticos, con la finalidad de mejorar el conocimiento, las estrategias o las actitudes y opiniones de los espectadores. En primer lugar, ha de poseer determinados valores o méritos humanos; en segundo lugar, dichos valores han de estar codificados de forma que sean interpretables y, en tercer lugar, que exista una intención de utilizar dicho medio como recurso formativo, es decir, con voluntad de producir algún tipo de aprendizaje, que no tiene por qué estar vinculado necesariamente al mensaje de la película» (De la Torre, 1996, citado por Pereira y Marín, 2001: 237).

De lo anterior se desprende que los docentes siguen siendo imprescindibles para poner el foco de la atención de los alumnos con una intención didáctica pertinente en cada caso, que suscite mediante la reflexión la ampliación del universo personal y colectivo.

\section{Desarrollo}

El proyecto fue diseñado para ser desarrollado dentro de Teoría e Historia de la Publicidad, asignatura anual y obligatoria, de 12 créditos, que se imparte en primer curso del Grado en Publicidad y RRPP y, por tanto, a alumnos recién llegados a la Universidad. Con él pretendíamos alcanzar los siguientes objetivos:

- Que los alumnos pudiesen comprender aspectos fundamentales del programa de la asignatura relativos al estudio de los fundamentos de la consolidación de la publicidad moderna.

- Permitir al alumnado comprender cuestiones esenciales de la Historia del siglo XX a través de la comprensión del desarrollo y evolución de la publicidad moderna.

- Extraer conclusiones de la aplicación práctica que nos permitiesen implementar herramientas para la docencia de la asignatura, que garantizasen la adquisición en el alumno de competencias recogidas en la Guía Docente, tales como:

- la capacidad para entender e interpretar el entorno y adaptarse al cambio

- el conocimiento del entorno económico, psicosocial, cultural y demográfico que les capacite para interactuar con la sociedad

- la capacidad y habilidad para utilizar las tecnologías y técnicas comunicativas, especialmente para aplicarlas al mundo de la comunicación, con especial énfasis en la creación de nuevos soportes, tales como las redes sociales online 
- la capacidad de relacionarse con las personas y con el entorno sin perder su autonomía, conservando su propia identidad y valores

- la capacidad para trabajar en equipo, desarrollando la apertura personal a través de la comunicación oral y escrita

- la capacidad de análisis, de síntesis y juicio crítico

En la primera fase se aplicó una metodología ya experimentada por las profesoras integrantes del proyecto a lo largo del curso 2010-11, en el marco de una investigación presentada en el I Congreso Nacional de Metodologías de Investigación en Comunicación (San Pablo y Pacheco, 2011). Con la práctica diseñada, basada en el visionado guiado de extractos de la primera temporada de la serie Mad Men, los alumnos tuvieron la oportunidad de asimilar contenidos relativos al desarrollo de la actividad publicitaria después de la $2^{\mathrm{a}}$ Guerra Mundial. Tras una introducción teórica acerca del marco histórico en el que se desarrolla la serie, los alumnos vieron dichos fragmentos, tomando notas para la posterior elaboración de un artículo escrito individualmente en el que debían relacionar los contenidos de lo visionado con la materia estudiada. Posteriormente, los alumnos trabajaron en grupos de cuatro o cinco personas para, a partir de sus visiones particulares, establecer una postura común que debía ser confrontada con la de otros grupos elegidos al azar en el aula. La práctica se completó con una serie de debates en clase para lograr extraer conclusiones colectivas.

En la segunda etapa del proyecto se diseñó la jornada «Para comprender la publicidad», que se celebró el 28 de noviembre de 2011 en la Facultad de Ciencias Sociales, Jurídicas y de la Comunicación de la Universidad de Valladolid. Con ella, los alumnos tuvieron la posibilidad de profundizar en el entorno de la serie, ahondando en los paralelismos entre los años 60 y el momento actual; su relevancia y pertinencia, así como la transformación del modelo publicitario-social que la serie Mad Men relata. En sesiones de mañana y tarde, tuvieron lugar una serie de conferencias y una mesa redonda, en las que expertos y teóricos de reconocido prestigio, tanto del ámbito profesional como del académico, ofrecieron a los alumnos distintas visiones del $f e$ nómeno Mad Men, enmarcadas en un análisis que contempló las implicaciones económicas, sociales y culturales así como la influencia de la publicidad en las sociedades capitalistas contemporáneas.

El título de la jornada se inspiraba en las relevantes aportaciones contenidas en la penúltima obra publicada del profesor Caro (2010), quien impartió la conferencia inaugural de la jornada. Antonio Caro es una figura imprescindible en el panorama académico latinoamericano, ya que su trabajo de investigación sobre la publicidad está contribuyendo a la consolidación de unos fundamentos epistemológicos para el estudio científico de esta disciplina ${ }^{2}$. Participaron igualmente en la jornada Gem Romero,

2 Antonio Caro lleva desde 1964 directamente relacionado con el fenómeno publicitario, primero como creativo profesional (fundador y codirector creativo de Contrapunto, elegido en varias ocasiones como uno de los redactores publicitarios más destacados de España) y, entre 1990 y 2009, como profesor de Creatividad Publicitaria y Teoría de la Publicidad en la Universidad Complutense de Madrid, labor docente que prosigue en la actualidad como profe- 
quien desde marzo de 2010 es Director Estratégico Creativo de la agencia de publicidad Bassat Ogilvy ${ }^{3}$, así como David Sánchez Usanos, profesor titular interino de Metafísica y Filosofía Contemporánea de la Universidad Autónoma de Madrid ${ }^{4}$.

La conjunción de las aportaciones de estos tres profesionales de primer nivel permitió enriquecer la perspectiva de análisis del fenómeno publicitario, al integrarse tres visiones complementarias: la de un académico con una dilatada y sólida trayectoria investigadora y profesional en el campo de la publicidad, la de un profesional en activo en la cúpula de una de las agencias de publicidad más relevantes del panorama mundial, así como la de un académico interesado en el estudio de la publicidad desde una disciplina esencial como es la filosofía.

Al término de la jornada, los alumnos hubieron de responder a un cuestionario de evaluación con el que se pretendía conocer el grado en que esta actividad había calado en ellos. Apelando a su capacidad de reflexión y a su sentido crítico, solicitábamos que plasmasen sus impresiones acerca de lo que ellos consideraban los principales contenidos expuestos por los ponentes, así como lo que dichos contenidos les habían suscitado. En base a los temas que más se repetían en sus respuestas, definimos un guión para realizar debates en el aula. Dichos debates, celebrados la semana posterior a la jornada, fueron moderados por los profesores de la asignatura y se articularon en torno a los siguientes conceptos e ideas:

- la publicidad como algo más que una herramienta de venta

- la configuración de la identidad a través de las marcas

- el hiperconsumo y la responsabilidad de la publicidad

sor invitado de diferentes universidades españolas y latinoamericanas. Ha publicado hasta el momento 7 libros y más de 50 artículos en publicaciones científicas y libros colectivos. Entre 2007 y 2011 ha sido codirector de Pensar la Publicidad. Revista Internacional de Investigaciones Publicitarias, editada por la Universidad Complutense de Madrid y la Universidad de Valladolid, de la que igualmente es fundador.

3 Con anterioridad a su incorporación fue Director General de Estrategia de TAPSA Madrid, Director Asociado en la consultoría Added Value PLANNERS y Strategic Planner en S, C, P, F., las tres compañías integrantes del Grupo WPP. Ha trabajado para clientes sectores muy diversos, tales como Vodafone, Caja Madrid, Banco de Santander, Mutua Madrileña, Iberia, Nike, Coca Cola, Unilever, Cola Cao, Nestlé, Panrico, San Miguel, Damm, Heineken, Pepsico, Castell de Perelada, Ausonia, Dodot, Cepsa, 11811, TVE, Gol Televisión, El País, Pans\&Company, Laboratorios Cinfa, Myrurgia, Planeta, Generalitat de Catalunya, Ajuntament de Barcelona y Comunidad de Madrid. Es profesor en distintas escuelas de negocios y universidades, tanto de Barcelona como de Madrid. Es socio fundador de la APG Spain y miembro de su junta directiva. También es miembro del Club de Jurados de los Premios Eficacia.

4 Además de haber investigado sobre la experiencia temporal (tanto en la Grecia antigua y clásica como en la postmodernidad), la presencia de elementos mágicos, míticos y rituales en la escritura y las relaciones entre filosofía y literatura, se ha ocupado de estudiar la publicidad desde una perspectiva filosófica y ha participado en la obra colectiva Guía de Mad Men. Reyes de la Avenida Madison. 
- la publicidad y el arte

- el anuncio como producto cultural de consumo

Además de la jornada mencionada, durante esta fase del proyecto se llevaron a cabo las siguientes actividades:

a) Trabajo en el aula. En el marco del programa teórico de la asignatura Teoría de la Publicidad se explicaron a los alumnos conceptos básicos y fundamentales para la comprensión de tres aspectos clave, a saber:

- La marca y su papel en la publicidad

- El contexto sociocultural de la publicidad

- La publicidad y sus actores: la agencia de publicidad

b) Visionado guiado. Para ayudar a los alumnos a profundizar en los conceptos teóricos expuestos en el aula, a principios de noviembre se proyectó en el aula el documental Comprar, tirar, comprar (Dannoritzer, 2011), un interesante documento acerca de la obsolescencia programada, sus causas e implicaciones en la economía actual. De esta forma se pretendía que los alumnos dispusieran de un sustrato informativo que les permitiera comprender algunos aspectos que serían tratados posteriormente en la jornada-seminario, particularmente las interrelaciones entre publicidad, consumo, sostenibilidad y ética.

c) Utilización de las TIC's. Aprovechando el manejo que tienen de las nuevas tecnologías, los alumnos hubieron de crear un hashtag que permitiese acceder y publicar comentarios desde la plataforma Twitter durante el desarrollo de la jornada «Para comprender la publicidad». Cabe destacar que la propuesta fue recibida con entusiasmo por los estudiantes y que a lo largo de la jornada se generaron varios cientos de tweets. Por otra parte, alumnos pertenecientes a la asociación estudiantil de la Facultad, Emisión Mahonita, grabaron en vídeo la jornada. Dicha grabación, una vez editada por los propios alumnos, se colgó en la plataforma Vimeo.com ${ }^{5}$ lo cual ha posibilitado que la difusión y aprovechamiento de dichos contenidos no se agotase con la celebración de la jornada sino que, yendo más allá del ámbito universitario, pudiesen ser consultados por cualquier persona interesada en «comprender la publicidad».

\section{Resultados y valoración del proyecto}

En la primera fase del proyecto se obtuvieron unos resultados satisfactorios a la luz del análisis de las primeras prácticas realizadas en el aula. Los estudiantes reconocieron en sus trabajos, en un elevado porcentaje, que el contacto con la serie Mad Men les había proporcionado herramientas muy concretas para hacer una inmersión en el contexto no sólo publicitario de la segunda mitad del siglo XX, sino también social y económico. Los estudiantes destacaron que les había ayudado a comprender los hábitos sociales de consumo en el mercado y los modos de producción capitalista sobre los que se ha cimentado la producción publicitaria en el mundo occidental contem-

5 Los vídeos de la jornada están disponibles para su visionado y descarga en http://vimeo.com/35128984, http://vimeo.com/34949107, http://vimeo.com/34954780. 
poráneo, así como los roles sociales desempeñados por hombres y mujeres, que tan marcadamente se exponen en la serie analizada.

Asimismo, hemos podido comprobar que los estudiantes asimilan de modo muy satisfactorio el contenido de la materia relativo a la organización de una agencia de publicidad moderna, así como la gestación de la publicidad en el seno de la neoyorquina Madison Avenue, epicentro de la actividad publicitaria moderna mundial. Es resaltable el hecho de que los alumnos de primer curso del Grado hayan hecho especial hincapié en sus reflexiones escritas en las relaciones sociales que marcan el inicio de la configuración del mercado de consumo y de la gestación de la publicidad modernos. Por otra parte, en los posteriores trabajos elaborados en grupo y en los debates desarrollados en el aula, hemos comprobado cómo los alumnos han comprendido que la época recreada en la serie, los años sesenta, es un momento matriz del cual se derivan importantes implicaciones, no sólo en lo estrictamente publicitario sino también en la configuración de las sociedades actuales.

Por ello podemos afirmar que se han satisfecho los objetivos que inicialmente nos proponíamos conseguir en cuanto a la utilización de la serie Mad Men como herramienta para la docencia de la asignatura Teoría e Historia de la Publicidad de primer curso del Grado en Publicidad y Relaciones Públicas.

En la segunda fase del proyecto hemos logrado profundizar en lo expuesto anteriormente, a través del desarrollo de actividades prácticas de visionado guiado, que han permitido al alumnado la adquisición de competencias generales y específicas en la Historia del siglo XX, así como la comprensión del desarrollo y evolución de la publicidad moderna. Los resultados obtenidos a raíz de la celebración de la jornada «Para comprender la publicidad» permiten constatar la aprehensión de contenidos de tres categorías muy específicas: por un lado, una de carácter teórico, que atañe a «pensar la publicidad» como un fenómeno que trasciende la mera promoción de los productos anunciados. Resulta muy significativo que alumnos tan jóvenes sean capaces de plantearse la influencia de la publicidad en la construcción de la imagen - tanto estética como social o, incluso, psicológica - de las personas. Por otro lado, un alto porcentaje de los alumnos han resaltado en sus trabajos el fenómeno del hiperconsumo como algo que les preocupa y con lo que se sienten concernidos, no sólo como consumidores sino también como futuros profesionales publicitarios. Por último, desde un punto de vista filosófico, sus reflexiones entroncan con aspectos éticos, que les hacen ser conscientes de que los valores sociales pasan, indefectiblemente, por valores económicos y políticos, con los cuales la publicidad (y los publicistas) deberían comprometerse.

Ciertamente, como docentes resulta muy gratificante comprobar que estudiantes tan jóvenes incorporan con tanta lucidez los más altos valores sociales y éticos para la construcción de una futura sociedad con aspiraciones de ser más justa que la actual. Consideramos que las herramientas experimentadas en este proyecto aportan a la docencia de la asignatura el cauce para que los alumnos se cuestionen con bastante profundidad para su formación y edad algunos de los retos que la publicidad tiene planteados hoy. Por otra parte, tales herramientas contribuyen a la consecución de los resultados de aprendizaje establecidos para la asignatura y recogidos en la Guía Do- 
cente de la misma. Así mismo, todo parece indicar que contribuyen fehacientemente al logro de las competencias que inicialmente nos planteábamos conseguir. Como ya hemos señalado más arriba, valoramos muy especialmente la capacidad que los alumnos han demostrado para asumir referentes éticos, valores y principios consistentes.

\section{Referencias bibliográficas}

APARICI, Roberto (2010): "La educomunicación más allá del 2.0", en APARICI, Roberto (ed.): Educomunicación: más allá del 2.0. Barcelona, Gedisa, pp. 9-23.

BAUMAN, Zygmunt (2007): Tiempos líquidos. Barcelona, Tusquets.

CARO ALMELA, Antonio (2010): Comprender la publicidad. Barcelona, Trípodos.

COLL, Mercé; SELVA, Marta; SOLÁ, Anna (1995): "Propuestas y orientaciones", Cuadernos de Pedogogía, $\mathrm{n}^{\circ}$ 242. Barcelona, Wolters Kluwer, pp. 14-33.

FERRÉS i PRATS, Joan (2010): “Educomunicación y cultura participativa”, en APARICI, Roberto (ed.): Educomunicación: más allá del 2.0. Barcelona, Gedisa, pp. 251-266.

KAPLÚN, Mario (2010): “Una pedagogía de la comunicación”, en APARICI, Roberto (ed.): Educomunicación: más allá del 2.0. Barcelona, Gedisa, pp. 41- 61.

PEREIRA DOMÍNGUEZ, $M^{\mathrm{a}}$ Carmen y MARÍN VALLE, M ${ }^{\mathrm{a}}$ Victoria (2001): "Respuestas docentes sobre el cine como propuesta pedagógica. Análisis de la situación en educación secundaria", Revista de Teoría de la Educación, no 13. Salamanca, Universidad de Salamanca, pp. 233- 255.

SAN PABLO MORENO, Pilar y PACHECO RUEDA, Marta (2011): “Aprender con el cine: una propuesta metodológica para la adquisición de competencias emocionales y audiovisuales", en 1er Congreso Nacional de Metodología de la Investigación en Comunicación: http://www.ucm.es/info/mdcs/Comunicaciones \%20Eje\%20Tematico\%202.pdf, [fecha de consulta: 11 de noviembre de 2012]

\section{$\overline{\text { Marta PACHECO RUEDA }}$}

Universidad de Valladolid, Campus María Zambrano (Segovia)

Facultad de Ciencias Sociales y Jurídicas y de la Comunicación

Profesora Contratada Doctora del área de Comunicación Audiovisual y Publicidad martapr@ega.uva.es 